\title{
Focal Autoimmune Pancreatitis: A Simple Flow Chart for a Challenging Diagnosis
}

\section{두)(1) 우우}

Authors

Clara Benedetta Conti, Fabrizio Cereatti, Andrea Drago, Roberto Grassia

\author{
Affiliation \\ Gastroenterology and Digestive Endoscopy Unit, ASST \\ Cremona, Cremona, Italy
}

Key words

pancreas, areas, structures \& systems, abdomen, areas, structures \& systems, endoscopy, methods \& techniques, ultrasound

received $\quad 15.09 .2020$

accepted after revision $\quad 25.11 .2020$

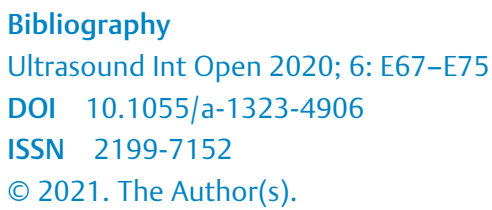

This is an open access article published by Thieme under the terms of the Creative Commons Attribution-NonDerivative-NonCommercial-License, permitting copying and reproduction so long as the original work is given appropriate credit. Contents may not be used for commercial purposes, or adapted, remixed, transformed or built upon. (https://creativecommons.org/ licenses/by-nc-nd/4.0/)

Georg Thieme Verlag KG, Rüdigerstraße 14,

70469 Stuttgart, Germany

\author{
Correspondence \\ Clara Benedetta Conti \\ Gastroenterology and Digestive Endoscopy, ASST Cremona, \\ Viala Concordia 1 \\ 26100 Cremona \\ Italy \\ Tel.: + 393496009047 \\ benedetta.conti1@gmail.com
}

\begin{abstract}
Autoimmune pancreatitis is a chronic fibroinflammatory autoimmune mediated disease of the pancreas. Clinically, obstructive painless jaundice and upper abdominal pain are the main symptoms. Focal AIP is characterized by segmental involvement of pancreatic parenchyma and it is often radiologically represented by a pancreatic mass. In these cases, the diagnosis can be very challenging, since it may be easily confused with pancreatic cancer. Therefore, we suggest a combined approach of imaging tests as the diagnostic workup. EUS study combined with CEUS and elastography, if available, increases the accuracy of the method to rule out cancer. Moreover, the lesion should always be sampled under EUS guidance to obtain a cyto/histological diagnosis. The diagnostic workup should also include the use of diagnostic clinical criteria (extrapancreatic lesions, steroid response) and laboratory findings (CA 19.9 and lgG4 evaluations).
\end{abstract}

\section{Introduction}

Autoimmune pancreatitis (AIP) is a chronic pancreatic fibroinflammatory autoimmune mediated disease. Obstructive painless jaundice and upper abdominal pain are the main symptoms. Many different classifications of AIP have been proposed: JPS $(2002,2006)$ [1], HISORt (2006, 2009) [2], Korean (2007) [3], Asian (2008) [4], Mannheim (2009) [5], Italian (2003, 2009) [6].

A review of these criteria led to the formulation of international consensus diagnostic criteria (ICDC) in 2011 [7], where two main AIP subtypes have been described ( $\vee$ Table 1 ).

Type 1 AIP: lymphoplasmacytic sclerosing pancreatitis, with dense periductal infiltration of plasma cells and lymphocytes, peculiar storiform fibrosis, venulitis with lymphocytes and plasma cells, obliteration of veins. Serologically, it shows abundant immunoglobulin (Ig)G4-positive plasma cells, as a pancreatic manifestation of IgG4-related systemic disease, with extrapancreatic lesions with infiltration of IgG4-positive plasma cells [8]. Some possible associations with AIP are sclerosing cholangitis, retroperitoneal fibrosis, lachrymal/salivary gland lesions, pulmonary hilar lymphadenopathy, tubulointerstitial nephritis, hypophysitis, chronic thyroiditis, prostatitis. Moreover, the response to steroid therapy is excellent (97-98\%) [9].

Type 2: Specific IgG4-negative pancreatic disease, with idiopathic duct-centric pancreatitis (IDCP) or AIP with granulocyte epithelial lesions (GELs) and lumen obliteration of medium, intraepithelial neutrophils, periductal lymphoplasmacytic infiltrate fibrosis. The prevalence of inflammatory bowel disease in patients with AIP is $30 \%$ [10].

Despite formulation of the ICDC with clinical/histological criteria, the diagnosis of AIP remains challenging: ICDC criteria are not internationally applied, impossibility of sampling, or technical difficulties in meeting histological criteria. Moreover, the spectrum of 
- Table 1 ICDC criteria for AIP diagnosis.

\begin{tabular}{|c|c|c|}
\hline & TYPE 1 AIP & \\
\hline CRITERION & LEVEL 1 & LEVEL 2 \\
\hline Parenchymal imaging [P] & $\begin{array}{l}\text { Typical } \\
\text { Diffuse enlargement with delayed enhancement (with or } \\
\text { without rim-like enhancement) }\end{array}$ & $\begin{array}{l}\text { Indeterminate (and atypical) } \\
\text { Segmental/focal enlargement with delayed } \\
\text { enhancement }\end{array}$ \\
\hline Ductal imaging (ERP) [D] & $\begin{array}{l}\text { Long ( }>1 / 3 \text { of the total length of } \mathrm{MD} \text { ) or multiple strictures } \\
\text { without marked upstream dilation }\end{array}$ & $\begin{array}{l}\text { Segmental/focal narrowing without marked upstream } \\
\text { dilation (duct size }<5 \mathrm{~mm} \text { ) }\end{array}$ \\
\hline $\begin{array}{l}\text { Serology [S] Other organ } \\
\text { involvement [OOI] }\end{array}$ & $\begin{array}{l}\text { IgG4 increased }>2 x \text { upper normal value } \\
\text { A or B } \\
\text { A: histology of extrapancreatic organs } \\
\text { (3 or more) } \\
\text { 1-marked lymphoplasmacytic infiltration with fibrosis and } \\
\text { without granulocytic infiltration } \\
\text { 2-storiform fibrosis } \\
\text { 3-obliterative phlebitis } \\
\text { 4-IgG4-positive cells >10/HPF } \\
\text { B: Typical radiological evidence } \\
\text { (one of) } \\
\text { 1-segmental/multiple proximal or proximal and distal bile } \\
\text { duct stricture } \\
\text { 2-retroperitoneal fibrosis }\end{array}$ & $\begin{array}{l}\text { IgG4 increased < } 2 \text { x upper normal value } \\
\text { A or B } \\
\text { A: histology of extrapancreatic organs + endoscopic } \\
\text { biopsies of bile duct } \\
(1+2) \\
1 \text {-marked lymphoplasmacytic infiltration without } \\
\text { granulocytic infiltration } \\
2 \text { - IgG4-positive cells }>10 / H P F \\
\text { B: physical or radiological evidence } \\
\text { (one of) } \\
\text { 1-symmetrically enlarged salivary or lachrymal glands } \\
\text { 2-radiological evidence of renal involvement }\end{array}$ \\
\hline Histology of pancreas [H] & $\begin{array}{l}\text { LPSP (biopsy/resection) } \\
\text { ( } 3 \text { or more) } \\
\text { 1-periductal lymphoplasmacytic infiltrate without granulocyt- } \\
\text { ic infiltration } \\
\text { 2-obliterative phlebitis } \\
\text { 3-storiform fibrosis } \\
\text { 4-IgG4-positive cells > 10/HPF }\end{array}$ & $\begin{array}{l}\text { LPSP (biopsy) } \\
\text { ( } 2 \text { of) } \\
\text { 1-periductal lymphoplasmacytic infiltrate without } \\
\text { granulocytic infiltration } \\
\text { 2-obliterative phlebitis } \\
\text { 3-storiform fibrosis } \\
\text { 4-IgG4-positive cells > 10/HPF }\end{array}$ \\
\hline \multirow[t]{3}{*}{ Response to steroid [Rt] } & $\begin{array}{l}\text { Diagnostic steroid trial: }<2 \text { weeks treatment with } \\
\text { radiological resolution or marked improvement in pancreatic } \\
\text { or extrapancreatic manifestations }\end{array}$ & \\
\hline & TYPE 2 AIP & \\
\hline & LEVEL 1 & LEVEL 2 \\
\hline Parenchymal imaging [P] & $\begin{array}{l}\text { Typical } \\
\text { Diffuse enlargement with delayed enhancement (with or } \\
\text { without rim-like enhancement) }\end{array}$ & $\begin{array}{l}\text { Indeterminate (and atypical) } \\
\text { Segmental/focal enlargement with delayed } \\
\text { enhancement }\end{array}$ \\
\hline Ductal imaging (ERP) [D] & $\begin{array}{l}\text { Long ( }>1 / 3 \text { of the total length of } \mathrm{MD} \text { ) or multiple strictures } \\
\text { without marked upstream dilation }\end{array}$ & $\begin{array}{l}\text { Segmental/focal narrowing without marked upstream } \\
\text { dilation (duct size }<5 \mathrm{~mm} \text { ) }\end{array}$ \\
\hline $\begin{array}{l}\text { Serology [S] } \\
\text { Other organ involvement [OOI] }\end{array}$ & & Inflammatory bowel disease \\
\hline Histology of pancreas [H] & $\begin{array}{l}\text { IDCP (resection (biopsy)) } \\
(1+2) \\
1 \text {-granulocytic infiltration of duct wall (GEL) with or without } \\
\text { granulocytic acinar inflammation } \\
2 \text {-IgG4 positive cells } 0-10 / \mathrm{HPF}\end{array}$ & $\begin{array}{l}\text { IDCP (resection (biopsy)) } \\
(1+2) \\
1 \text {-granulocytic and lymphoplasmacytic acinar } \\
\text { infiltrate } \\
2 \text {-IgG4-positive cells } 0-10 / \text { HPF }\end{array}$ \\
\hline Response to steroid [Rt] & $\begin{array}{l}\text { Diagnostic steroid trial: }<2 \text { weeks treatment with } \\
\text { radiological resolution or marked improvement in pancreatic } \\
\text { or extrapancreatic manifestations }\end{array}$ & \\
\hline
\end{tabular}

clinical presentation is very broad ( $\triangleright$ Table 2 ) including the presence of a pancreatic mass.
Indeed, the radiological features of AIP range from normal pancreas to diffuse parenchymal enlargement with a "sausage-like" appearance, to a focal mass-like image. The presence of the latter 
- Table 2 AIP clinical presentations.

\begin{tabular}{|l}
\hline Clinical findings \\
\hline Jaundice \\
\hline Mild abdominal pain \\
\hline Endocrine insufficiency (diabetes) \\
\hline Weight loss \\
\hline Persistent hyperamylasemia \\
\hline Recurrent episodes of acute pancreatitis of unknown origin \\
\hline Pancreatic mass or pancreatic enlargement incidentally found at imaging \\
\hline One of the criteria above and concomitant other organ involvement \\
\hline
\end{tabular}

radiological appearance is an indication of focal AIP (f-AIP) [11, 12]. Mainly, the focal type appears on imaging as a focal mass with blurred outlines. Dilation of the main pancreatic duct can be evident and the image can be easily confused with a neoplastic pancreatic lesion [11]. Conversely, the other imaging presentations are suggestive of diffuse forms of AIP.

This review aims to describe the available tests to better diagnose focal AIP, ruling out pancreatic cancer (PC) and giving a possible effective diagnostic approach.

\section{Focal autoimmune pancreatitis}

F-AIP is characterized by segmental involvement of the pancreatic parenchyma and it is radiologically represented by a pancreatic mass. The literature does not report precise data on the prevalence of the focal form in Type I or II pancreatitis. However, Type I f-AIP seems to definitely be more frequent than Type II [12]. Diagnosis can be very challenging, as it may be easily confused with PC. Since $\mathrm{f}$-AIP is a benign condition that is dramatically responsive to steroid therapy within one month in $90 \%$ of cases [7], it is mandatory to histologically rule out cancer, thereby avoiding pancreatic surgery. Currently, the only reference standard for diagnosis of F-AIP is the surgical specimen. Moreover, the prevalence of PC in the general population is much higher than that of AIP. It is the fourth leading cause of cancer-related fatalities in Western countries. Therefore, early treatment is crucial for achieving cure. Unfortunately, the clinical incidence of $\mathrm{f}$-AIP among all AIP cases is unknown. The only available data relate to f-AIP cases diagnosed as cancer.

Several studies reported large series of misdiagnosed f-AIP that was surgically treated. In 2003, Abraham et al. [13] reported that $10.6 \%$ of Whipple resections among 442 pancreaticoduodenectomies were negative for neoplasia, with $12.8 \%$ being chronic pancreatitis (CP) of unknown etiology, and $23.4 \%$ being AIP 1 . In a different surgical series [14] the incidence of CP was $13 \%(21 / 162$ specimens). Another two series reported benign pathology in $7 \%$ [15] and $23 \%$ [16] of cases among patients who underwent surgery for cancer. The lack of accurate markers to differentiate between PC and non-malignant pancreatic lesions, such as blood test or other noninvasive tools with a high positive predictive value, is the main reason behind diagnostic failures. Thus, the first goal regarding pancreatic masses is to definitively rule out the presence of cancer, even if clinical and serological findings (high level of $\operatorname{lgG} 4$, presence of autoantibodies, low serum levels of Ca 19-9) are suggestive for AIP [17].

\section{Diagnosis of focal AIP}

\section{Serum markers}

Serum lgG4 levels may rise to twice the normal value in AIP 1. However, IgG4 elevation may also be present in PC. When IgG4 serum levels were examined in 115 patients with cancer, plasmatic IgG4 levels were higher than normal in 14 patients and double in 2 patients. One case had an overlap diagnosis between f-AIP and PC. No larger quantity of data about overlap between f-AIP and PC is available. Serum IgG4, CEA, and CA19-9 levels were measured in 188 patients [18]. A combined use of serum $\mathrm{lgG} 4$ (over $280 \mathrm{mg} / \mathrm{dL}$ ) and CA19-9 9 (below $85.0 \mathrm{U} / \mathrm{ml}$ ) was suggested to increase the diagnostic accuracy to distinguish AIP from PC. When using an IgC cutoff value of $175 \mathrm{mg} / \mathrm{dL}$, the sensitivity and specificity for differential diagnosis were 67.5 and $90.4 \%$, respectively [19]. However, these data have weak evidence, and the diagnosis of PC versus AIP cannot be made only using serological parameters.

\section{Imaging}

\section{Transabdominal Ultrasound (US)}

US is usually the first diagnostic method performed in patients with jaundice or abdominal pain, because of its low cost and wide availability. However, the ability of US to detect pancreatic masses is related to operator experience and is reduced by the possible presence of bowel gas or obesity, due to the retroperitoneal pancreas location. Despite compression to displace bowel gas, asking for inspiration/expiration, changing the patient's position, US sensitivity, specificity, and accuracy range from 48-95, 40-91 and 46-64\%, respectively [20]. In a multicenter retrospective study, US detected the tumor in $52.6 \%$ of 135 cases of early-stage PC. Data about cancer screening in Japan showed that US detected less than $0.01 \%$ of cases of PC. [21]. Thus, US cannot be considered the reference standard for the study of PC and its limitations contraindicate US sampling. Differentiation between f-AIP and PC is even more difficult. The use of contrast-enhanced US (CEUS) and elastography (EG) may help. In a meta-analyses, the pooled sensitivity and specificity in the differential diagnosis between pancreatic adenocarcinoma (ADK) and other pancreatic masses with CEUS were 86-90 and $75-88 \%$, respectively [22]. Among 123 pancreatic lesions, the difference in stiffness between ADK and the normal pancreas was statistically significant ( $p$ 0.05) [23]. However, another study performed ARFI elastography in 27 solid pancreatic lesions: 8 benign (focal pancreatitis and AIP) and 19 malignant. No statistical difference was found. Therefore, US can be considered as a first-line test. CEUS can help in studying pancreatic masses, but it cannot be considered a good test for discriminating PC from f-AIP [24].

\section{Computed Tomography (CT scan)}

Regarding the role of multiphase contrast-enhanced (CE) CT for differentiating f-AIP from carcinoma, 22 f-AIP lesions and 61 malignant lesions were examined [25]. The frequencies of radiological findings between f-AIP and cancer were compared. At multivariate analysis dotted enhancement, the duct-penetrating sign and capsule-like rim were statistically significant for the diagnosis of AIP versus PC. The combination of these findings permitted AIP di- 
agnosis with $82 \%$ sensitivity and $98 \%$ specificity [26]. However, in another study [27] (32 pancreatic lesions), CT scan showed an accuracy of only $68 \%$ in the diagnosis of AIP. In addition, the agreement between radiologists with respect to distinguishing between benign and malignant masses seemed fair ( $\kappa, 0.58 ; \mathrm{p}<0.0001)$. Hence, based on the minimal available evidence, CT scan is a fair method for distinguishing between PC and f-AIP. To date, no large cohorts have been investigated.

Fluorodeoxyglucose Positron Emission Tomography (FDG-PET) The role of 18F-FDG PET/CT in distinguishing between f-AIP and PC was examined in 26 AIP and 40 PC patients. All 26 patients with AIP had increased pancreatic FDG uptake. The standardized uptake values (SUV) max in AIP patients were higher compared with those in PC patients $(p<0.05)$. However, the diagnostic sensitivity of SUV in the PC group was only $70 \%$. Furthermore, a quite remarkable metabolism was detected in some patients with AIP, leading to false positivity [28]. In a Chinese study [29], the sensitivity, specificity, and accuracy of $18 \mathrm{~F}-\mathrm{FDG}$ PET/CT in differentiating PC from f-AIP were 95,60 , and $83.3 \%$, respectively. Another retrospective analysis of 232 patients [30] showed that FDG-PET was not effective in detecting early stage PC or in differentiating f-AIP from PC. Therefore, FDG-PET is considered a poor method for distinguishing between f-AIP and PC.

\section{Magnetic Resonance Imaging (MRI)}

Regarding MRI, a study [31] examined 36 patients with f-AIP and 72 patients with $P C$ who underwent CE-MRI with triple phases. Quantitative analysis of the lesion contrast using CE-MRI was helpful to differentiate f-AIP from PC. For AIP, the sensitivity and specificity of the contrast arterial phase were 94.4 and $87.5 \%$, respectively, (LR + 7.55, LR- 0.06) and were comparable or significantly higher than those of all key imaging features. For PC, the sensitivity $(87.5 \%)$ and specificity $(94.4 \%)$ of the contrast arterial phase were comparable or significantly higher than those of all key imaging features, except for the discrete mass. Moreover, one study [32] retrospectively evaluated the combination of triple-phase CT scan of 79 patients ( 19 with f-AIP, 30 with PC, and 30 with a normal pancreas) with MRI findings. The diagnostic performance of CT attenuation changes from the arterial phase to the hepatic phase was significantly higher in f-AIP than in PC $(p<0.05)$, with a sensitivity, specificity, and area under the ROC curve of $87.5 \%, 100 \%$ and 0.974 (95\% Cl:0.928-1.021), respectively. Analysis of the combination of focal pancreatic enlargement with a capsule-like rim, irregular narrowing of the MPD, and stricture of the CBD in patients with lesions (not located in the pancreatic head) helped to improve the diagnostic accuracy for f-AIP. Conversely, the retrospective analysis of 22 patients [33] found when analyzing CT scans and MRI images that the diagnostic performance of combined unenhanced and CE-MR images was significantly better than that of CT $(p<0.01)$. These data were confirmed also in another retrospective cohort of 187 patients [34]. However, no studies prospectively evaluated in large cohorts the accuracy of either MRI alone or combined with $\mathrm{CT}$, to consistently exclude PC in the case of a pancreatic mass. Hence, the sensitivity and specificity of MRI are good. However, since the available studies are all retrospective with small samples, it is difficult to give external validity of their results and use MRI as the reference standard in differential diagnosis between $P C$ and f-AIP. However, MRI should be used to confirm the final diagnosis, and it could be considered in the follow-up of patients.

\section{Endoscopic Ultrasound (EUS)}

Endoscopic ultrasound (EUS) is nowadays widely available and provides high-resolution images of the pancreas without interference from bowel gas. It is an invasive technique, but it does not expose the patient to radiation, allows the use of a contrast medium almost free of side effects, and enables direct guided sampling of pancreatic masses. No EUS imaging features are described as pathognomonic of f-AIP or PC. However, some signs could be helpful in the differential diagnosis ( $>$ Fig. 1): presence or absence of macroscopic vascular invasion, extrapancreatic local spread of the mass, and presence of pancreatic duct dilation. When vascular or

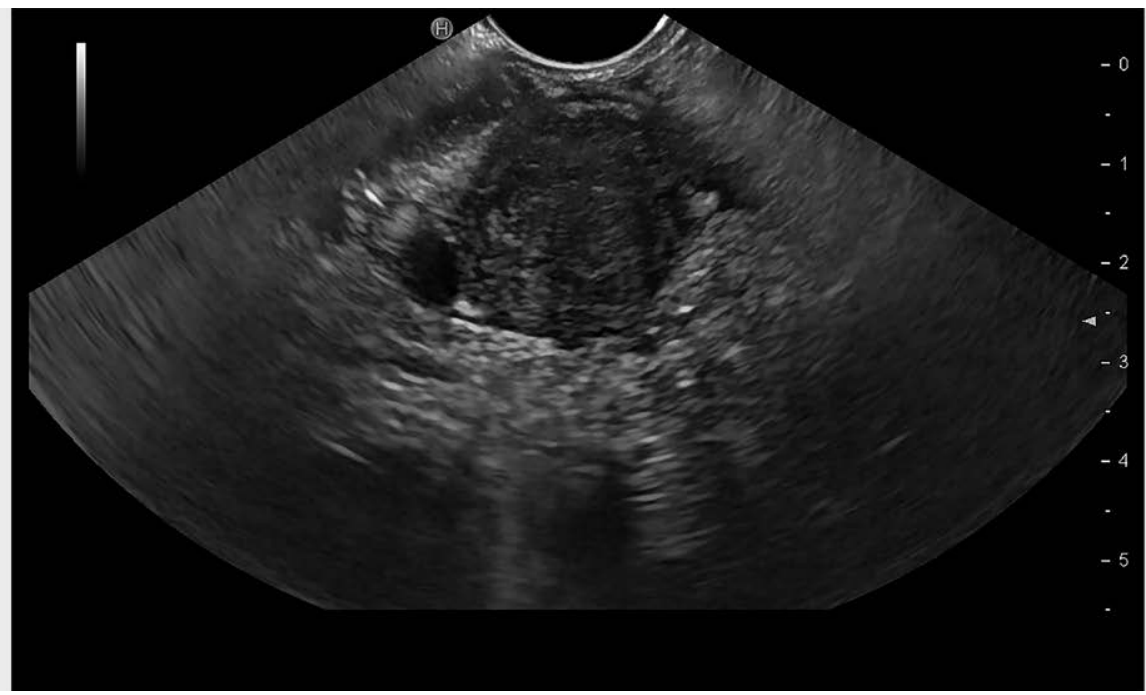

- Fig. 1 EUS image of a focal pancreatic lesion. 
extrapancreatic invasion is clear, the diagnosis of PC can be quite easy. However, EUS can also detect the apparent involvement of the portal and/or superior mesenteric vein in AIP when the inflammatory infiltrate transmurally involves the vessel wall [35]. In the case of well-differentiated ADK appearing as a small lesion with no clear vascular invasion, the differentiation between benign and malignant lesion can be hard. It is exactly in these cases that, if f-AIP is present, ruling out $P C$ with a high level of certainty is mandatory in order to avoid surgery. The application of the Rosemont criteria ( $\triangleright$ Table 3) for the surrounding pancreatic parenchyma around the focal mass could be useful. However, EUS alone has shown slightly disappointing accuracy for differentiating PC from CP (i. e., 76\% for malignancy and $46 \%$ for focal inflammation) [36].

\section{FNA/FNB sampling}

EUS-guided sampling should always be performed in the case of pancreatic solid lesions. FNA has a diagnostic accuracy ranging from 77-95\% [37, 38], especially if coupled with on-site pathological evaluation (ROSE) $[39,40]$. It is a safe technique, with morbidity and mortality rates $<1 \%$. However, FNA is often unable to obtain core tissue with a preserved architecture and ROSE is mainly unavailable. Indeed, the current European Society of Gastrointes-

- Table 3 Rosemont criteria for the diagnosis of chronic pancreatitis.

\begin{tabular}{|l|l|}
\hline Parenchymal features & Ductal features \\
\hline Hyperechoic foci with shadowing & MPD calculi \\
\hline Lobularity with honeycombing & Irregular MPD \\
\hline $\begin{array}{l}\text { Lobularity without honeycombing } \\
\text { Hyperechoic foci without } \\
\text { shadowing }\end{array}$ & Dilated side branches \\
\hline Cysts & MPD dilation \\
\hline Stranding & Hyperechoic MPD margin \\
\hline
\end{tabular}

tinal Endoscopy guidelines suggest, if ROSE is unavailable, to perform three to four needle passes with an FNA needle or two to three passes with an FNB needle. AIP may mimic malignancy presenting the following cytological features: occasional atypical cells, large nuclei, degenerative vacuoles, sparse mitosis. Conversely, cells in AIP tend to lack hyperchromasia, display only minimal architectural disorders, and have only modestly increased nuclear-to-cytoplasmic ratios [40]. Therefore, cytology can be inconclusive. Hence, theoretically, a core biopsy with an FNB needle yields larger specimens, providing better samples with intact histological architecture, to rule out PC. The available needles range from a diameter of $19 \mathrm{G}$ to $25 \mathrm{G}$. Strong evidence is still lacking, but the literature shows some encouraging results. 25 G FNB seems to guarantee a higher amount of diagnostic cellular material and better preservation of the tissue architecture than 22 G FNA ( $p=0.030$ and 0.010 , respectively), with a better diagnostic yield for specific tumor discrimination ( $p=0.018$ ). In the absence of ROSE, the $20 \mathrm{G}$ FNB needle outperforms the $25 \mathrm{G}$ FNA needle in terms of histological yield ( 77 vs $44 \% ; P<0.001$ ) and diagnostic accuracy ( 87 vs. $78 \%$; $P=0.002$ ), with a $99 \%$ technical success rate for the FNB needle. [40]. Again, it was reported that using EUS trucut biopsy for acquiring core specimens and preserving tissue architecture could enhance the diagnostic accuracy for f-AIP [41]. Moreover, in a retrospective study [42], FNB reached higher diagnostic accuracy than FNA in distinguishing between inflammatory masses and PC (93 vs. $83.6 \%, p=0.03)$. F-AIPs were also included in this cohort. Therefore, FNB should be considered the preferred sampling technique to rule out cancer in patients with underlying $C P$, including $f$-AIP.

\section{Contrast-Enhanced Ultrasound (CEUS)}

SonoVue is a second-generation microbubble contrast agent used for the characterization of the microvascularization of a lesion to make a differential diagnosis between benign and malignant diseases (SonoVue, $4.8 \mathrm{ml}$ intravenous administration). EUS-US mode for CEUS allows dynamic observation. This clarifies the behavior of the lesion in the arterial and venous phases. Indeed, ADK has a typical

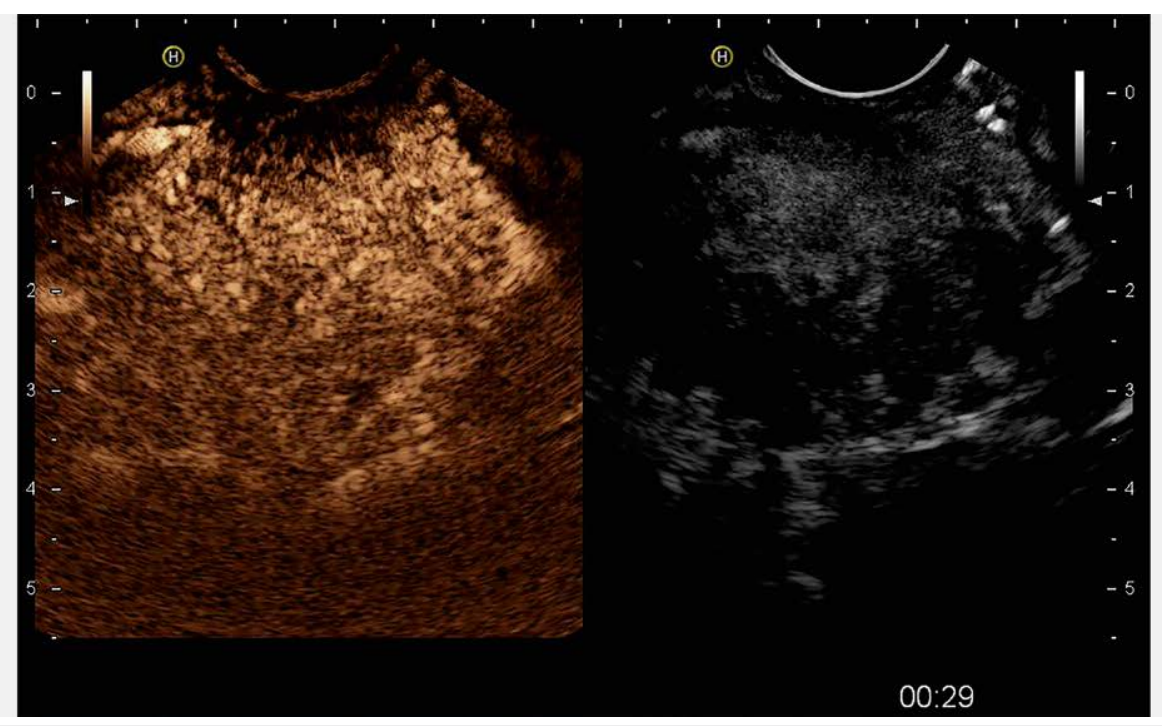

Fig. 2 CEUS study of focal autoimmune pancreatitis: iso-hyperenhancement of the mass. 
hypoenhancement in all phases. Conversely, neuroendocrine tumors show strong arterial hyperenhancement. Mass-forming CP and f-AIP have an isovascular or weak hypervascular appearance, similar to the surrounding pancreatic parenchyma ( $\mathbf{F i g}$. 2). These features may help in excluding PC. Indeed, in a retrospective data collection [39] including 60 cases of f-AIP and 16 cases of PC, $86.6 \%$ of AIP lesions displayed focal or diffuse isoenhancement in the arterial phase, while $93.7 \%$ of $P C$ lesions were hypoenhancing $(P<0.01)$. During the late phase, $65 \%$ of AIP lesions were hyperenhancing and $35 \%$ were isoenhancing, while $93.7 \%$ of PC cases were hypoenhancing. A retrospective study [43] investigated 80 patients diagnosed with f-AIP (27 patients) or $\mathrm{PC}$ (53 patients). Hyperenhancement to isoenhancement in the arterial phase ( $\mathrm{f}$-AIP 89 vs. PC $13 \%$; $p<0.05$ ), homogeneous contrast agent distribution (f-AIP 81 vs. PC $17 \%$; $p<0.05$ ), and absent irregular internal vessels (f-AIP 85 vs. PC $30 \%$; $p<0.05$ ) were observed more frequently in the f-AIP group. The combination of these features improved the specificity (94\%) for differentiating f-AIP from PC. Moreover, the overall diagnostic accuracy for CEUS was 83.33 vs. $44.4 \%$ for EUS only. ( $p<0.001)$. Importantly, the interobserver agreement for CEUS was significantly higher than that for US alone [44]. Interestingly, although only in a small series (3 AIP versus $17 \mathrm{PC})$, CEUS perfusion parameters were quantitatively analyzed with VueBox ${ }^{\circledR}$ quantification software. Significant differences between $P C$ and parenchyma could be found in terms of peak enhancement $(P E)$, wash-in and wash-out AUC, and wash-in perfusion index. The PE of AIP was comparable to that of a normal pancreatic parenchyma. The PE of PC was significantly lower than that of AIP or normal parenchyma $(p<0.01)$ [45]. In conclusion, although investigated in small cohorts, CEUS seems to increase EUS accuracy in the differential diagnosis between f-AIP and PC.

\section{Elastography}

Some studies investigated the role of EG in diagnosing f-AIP or other focal pancreatitis versus PC. One of the most popular commercially available EUS-EG techniques is real-time EG ( Fig. 3): a strain meth- od with a color scale. The operator evaluates this scale qualitatively during a routine EUS session. Blue and green colors indicate a stiffer tissue, while a red color indicates a less stiff tissue. However, the software can also measure the ratio between the target zone (lesion) and normal surrounding parenchyma. It therefore also provides a semiquantitative result. Cancers often present a higher stiffness value versus normal tissue or inflammation. Values can be expressed in $\mathrm{kPa}$ or in velocity of the wave [46]. A study measured the stiffness of 123 lesions (78 PC cases and $45 \mathrm{f}$-AIP cases). The strain ratio lesion/surrounding parenchyma correlated significantly with malignancies [47]. Similarly, a prospective study (325 patients) investigated the role of real-time EG in the differential diagnosis between benign (CP and AIP) versus malignant nodules. For the strain ratio lesion/parenchyma, a cut-off value of 4.2 versus 10.9 had a sensitivity, specificity, PPV, NPV, accuracy of $95,63,89,81$, and $87 \%$, respectively, versus $75,88,95,54$, and $79 \%$, respectively [48]. In another study with 9 cases of AIP, 40 cases of CP, and 130 cases of PC [49], EG had a sensitivity of $99 \%$, a specificity of $63 \%$, and an accuracy of $88 \%$. The best cut-off level of strain ratio to obtain the maximal ROC curve was 7.8 (accuracy of $88 \%$ ). Notably, in a meta-analysis that included 17 studies (1544 lesions), the pooled sensitivity and specificity for qualitative EG were 0.97 (95\% Cl, 0.95-0.99) and 0.67 (95\% $\mathrm{Cl}, 0.59-0.74)$, respectively; the pooled sensitivity and specificity for strain ratio were $0.98(95 \% \mathrm{Cl}, 0.96-0.99)$ and 0.62 (95\% Cl, 0.56$0.68)$, respectively; the pooled sensitivity and specificity for CEUS were $0.90(95 \% \mathrm{Cl}, 0.83-0.95)$ and $0.76(95 \% \mathrm{Cl}, 0.67-0.84)$, respectively; and the pooled sensitivity and specificity for EUS-FNA were $0.84(95 \% \mathrm{Cl}, 0.77-0.90)$ and $0.96(95 \% \mathrm{Cl}, 0.88-1.00)$, respectively. These results suggest a very similar sensitivity and specificity for EUS-EG and CEUS and they may be complementary studies for EUSFNA [50].

\section{Comparison of the techniques}

Based on previous observations regarding the accuracy of imaging in distinguishing between f-AIP and PC, the advantage of the CT

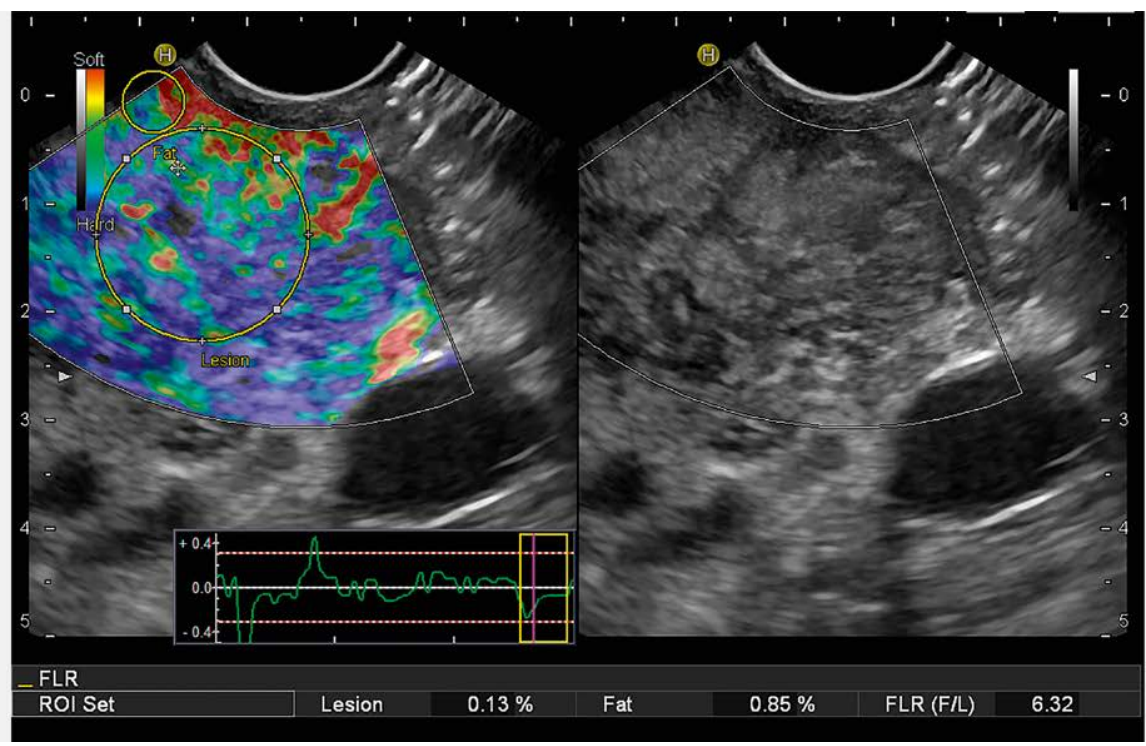

- Fig. 3 Real-time elastography study of focal autoimmune pancreatitis. 
- Table 4 Advantages and disadvantages of the imaging techniques to rule out cancer and diagnose f-AIP

\begin{tabular}{|c|c|c|}
\hline Imaging technique & Advantages & Disadvantages \\
\hline Transabdominal US & $\begin{array}{l}\text { - Broad availability } \\
\text { - Low cost } \\
\text { - Availability of CEUS, in case of good visibility of the } \\
\text { pancreatic mass }\end{array}$ & $\begin{array}{l}\text { - Very low accuracy in diagnosing PC and in differential } \\
\text { diagnosis PC versus f-AIP } \\
\text { - Operator-dependent }\end{array}$ \\
\hline CT scan & $\begin{array}{l}\text { - Broad availability } \\
\text { - Combination of more elements in the study of pancreatic } \\
\text { masses }\end{array}$ & $\begin{array}{l}\text { Poor interobserver agreement in the diagnosis of } \\
\text { pancreatic masses } \\
\text { - Low accuracy in some studies } \\
\text { - Radiation }\end{array}$ \\
\hline MRI & $\begin{array}{l}\text { - High sensitivity and specificity for diagnosis of PC in } \\
\text { retrospective cohorts } \\
\text { - Radiation-free } \\
\text { - Can be used in follow-up }\end{array}$ & $\begin{array}{l}\text { - Lack of large and prospective studies on accuracy in } \\
\text { distinguishing between PC and f-AIP }\end{array}$ \\
\hline FDG-PET & - No relevant advantages & - Very low accuracy in distinguishing between PC and f-AIP \\
\hline EUS & $\begin{array}{l}\text { - Good visibility of pancreatic masses } \\
\text { - High accuracy in ruling out PC } \\
\text { - Availability of CEUS and EG } \\
\text { - Sampling of the mass to rule out cancer }\end{array}$ & $\begin{array}{l}\text { - Invasive technique } \\
\text { - Operator-dependent }\end{array}$ \\
\hline
\end{tabular}

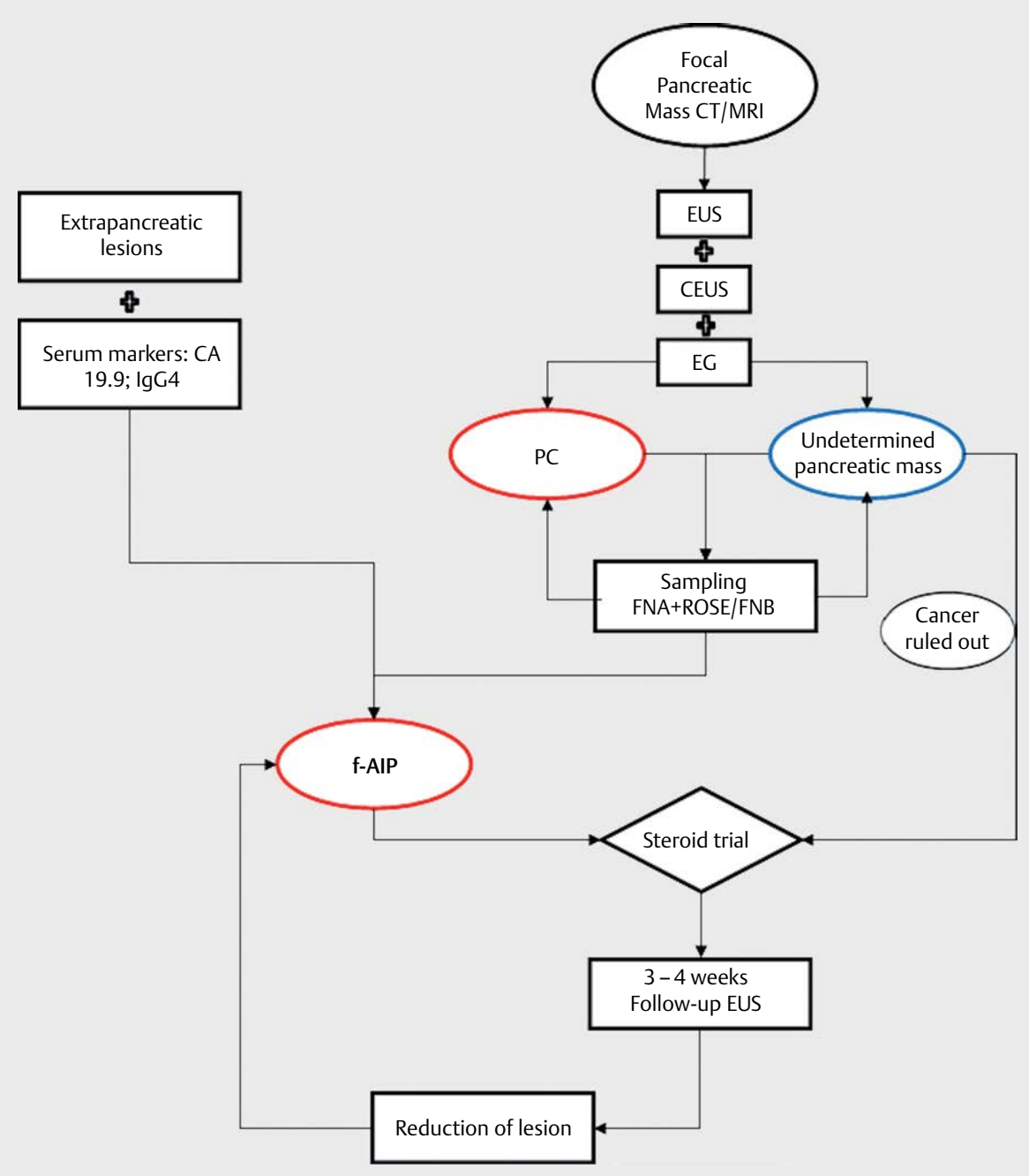

- Fig. 4 Diagnostic flowchart for focal autoimmune pancreatitis. 
scan is the availability of combining more elements in the study of the lesion, but some studies reported very low accuracy of CT and the lack of a good agreement between radiologists. The advantage of MRI is the high sensitivity and specificity of the contrast arterial phase study in the differential diagnosis of f-AIP versus PC. However, the lack of prospective studies is a relevant bias with regard to trusting the accuracy of the method as a reference standard. FGDPET has the lowest sensitivity, specificity, and accuracy among the imaging techniques. Therefore, neither advantages of using FDGPET alone in the diagnosis of $\mathrm{f}$-AIP versus PC were described, nor are studies combining MRI and/or CT with FDG-PET available. US can be used as the first screening modality but cannot be considered the reference test to study pancreatic masses or to differentiate PC from f-AIP, due to its low accuracy in detecting cancer and characterizing masses. EUS has the advantage of good evaluation of the pancreas in all patients, with the best sensitivity, specificity, and accuracy among the imaging tests with respect to detecting pancreatic masses and distinguishing between PC and f-AIP. The main advantage of EUS over US is the possibility of ruling out cancer combining B-mode, CEUS, elastography and sampling the mass. The best sensitivity, specificity, and accuracy reported in the studies for the diagnosis of PC for each technique are: 48-95, 40-91, and $46-64 \%$, respectively, for US; 77-80, 89-100, and 70-73\%, respectively, for CT scan; 98,97 , and $90 \%$, respectively, for EUS; $85-$ 90, 96-98, and 85-100\%, respectively, for EUS-FNA; 83-92, 63-89\%, respectively, for MRI [20-24]. > Table 4 summarizes the advantages and disadvantages of the imaging methods.

\section{Discussion: A Practical Flowchart to Diagnose F-AIP}

The diagnosis of $\mathrm{f}$-AIP can be very challenging. Currently, the only reference standard for the differential diagnosis between f-AIP and cancer is histological examination after surgery. Among the imaging tests, a reference standard for a definitive diagnosis is lacking. A combined approach with imaging and sampling of the lesion increases the diagnostic accuracy, helping to rule out cancer. Based on these observations, we propose the following diagnostic approach.

If a focal pancreatic mass is detected by means of CT scan or MRI, EUS should always be performed and combined with CEUS study and EG if available. When sampling a suspected pancreatic cancer, EUS-FNA sampling is recommended as the first-line procedure [37]. The lesion should be sampled with FNA needles if ROSE is available or with FNB in the absence of ROSE to obtain a core histology. This method seems to reduce the inconclusive diagnosis and better rule out cancer $[39,40]$. The diagnostic workup should also include: clinical examination, detection of possible extrapancreatic lesions, and CA 19.9 and IgG4 evaluation, in order to meet the clinical ICDC criteria if possible.

If $\mathrm{f}$-AIP is diagnosed, steroid treatment can be started. If $\mathrm{f}$-AIP cannot be diagnosed but cancer can be confidently ruled out, steroid treatment should be started and the patient should be strictly followed up. EUS can be performed again after 3-4 weeks, since this is the time estimated to obtain response after steroid treatment. In the case of AIP, the radiological features should change and if the diagnosis of AIP is correct, the second EUS examination should find an impressive reduction of the "lesion". We recommend using EUS for the reported good accuracy with respect to distinguishing between f-AIP and PC and, importantly, for the possibility of a second sampling [37] (if the first histology was inconclusive/ negative for cancer, but the mass does not reduce). However, MRI can be also considered for follow-up. The diagnostic flowchart for f-AIP diagnosis is presented in $>$ Fig. 4.

\section{Conflict of Interest}

The authors declare that they have no conflict of interest.

\section{References}

[1] Members of the Criteria Committee for Autoimmune Pancreatitis of the Japan Pancreas Society Diagnostic criteria for autoimmune pancreatitis by the Japan Pancreas Society (2002). J Jpn Pancreas Soc (Suizou) 2002; 17: 585-587

[2] Chari ST, Takahashi N, Levy M] et al. A diagnostic strategy to distinguish autoimmune pancreatitis from pancreatic cancer. Clin Gastroenterol Hepatol 2009; 7: 1097-03. PMID:19410017

[3] Kwon S, Kim MH, Choi EK. The diagnostic criteria for autoimmune chronic pancreatitis: it is time to make a consensus. Pancreas 2007; 34: 279-286. PMID: 17414049

[4] Otsuki M, Chung JB, Okazaki K et al. Asian diagnostic criteria for autoimmune pancreatitis: consensus of the Japan-Korea Symposium on Autoimmune Pancreatitis. J Gastroenterol 2008; 43: 403-408. PMID: 18600383

[5] Schneider A, Lohr JM. [Autoimmune pancreatitis]. Internist (Berl) 2009; 50: 318-330. PMID: 19212732

[6] Pearson RK, Longnecker DS, Chari ST et al. Controversies in clinical pancreatology: autoimmune pancreatitis: does it exist? Pancreas. 2003; 27: 1-13. PMID:12826899

[7] Shimosegawa T, Chari ST, Frulloni L et al. "International consensus diagnostic criteria for autoimmune pancreatitis: Guidelines of the international association of pancreatology,". Pancreas 2011; 3: 352-358. PMID: 21412117

[8] Okazaki K, Uchida K. Current concept of autoimmune pancreatitis and IgG4-related disease. Am J Gastroenterol 2018; 113: 1412-1416. PMID: 30002467

[9] Kamisawa T, Zen Y, Pillai S et al. IgG4-related disease. The Lancet 2015; 385: 1460-1471. PMID: 25481618

[10] Okazaki K, Kawa S, Kamisawa T et al. Japanese consensus guidelines for management of autoimmune pancreatitis: I. Concept and diagnosis of autoimmune pancreatitis. J Gastroenterol 2010; 45: 249-26. PMID: 20084528

[11] Wakabayashi T, Kawaura Y, Satomura Y et al. Clinical and imaging features of autoimmune pancreatitis with focal pancreatic swelling or mass formation: comparison with so-called tumor-forming pancreatitis and pancreatic carcinoma. Am J Gastroenterol 2003; 98: 26792687. PMID: 14687817

[12] Weber SM, Cubukcu-Dimopulo O, Palesty JA et al. Lymphoplasmacytic sclerosing pancreatitis: inflammatory mimic of pancreatic carcinoma. J Gastrointest Surg 2003; 7: 129-137. PMID: 12559194

[13] Abraham SC, Wilentz RE, Yeo C] et al. Pancreaticoduodenectomy (Whipple resections) in patients without malignancy: Are they all 'chronic pancreatitis'? Am J Surg Pathol 2003; 27: 110-120. PMID: 12502933

[14] Kennedy T, Preczewski L, Stocker S] et al. Bell et al. Incidence of benign inflammatory disease in patients undergoing Whipple procedure for clinically suspected carcinoma: a single-institution experience. Am J Surg 2006; 191: 437-441. PMID: 16490563 
[15] Hurtuk MG, Shoup M, Oshima K et al. Pancreaticoduodenectomies in patients without periampullary neoplasms: lesions that masquerade as cancer. Am J Surg 2010; 199: 372-376. PMID: 20226913

[16] De la Fuente SG, Ceppa EP, Reddy SK et al. Incidence of benign disease in patients that underwent resection for presumed pancreatic cancer diagnosed by endoscopic ultrasonography (EUS) and fine-needle aspiration (FNA). J Gastrointest Surg 2010; 14: 1139-1142. PMID: 20424928

[17] Frulloni L, Amodio A, Katsotourchi AM et al. A practical approach to the diagnosis of autoimmune pancreatitis. World J Gastroenterol 2011; 17: 2076-2079. PMID: 21547125

[18] Dite P, Novotny I, Dvorackova J et al. Pancreatic solid focal lesions: differential diagnosis between autoimmune pancreatitis and pancreatic cancer. Dig Dis 2019; 37: 416-442. PMID: 31079114

[19] Chang MC, Liang PC, Jan S et al. Increase diagnostic accuracy in differentiating focal type autoimmune pancreatitis from pancreatic cancer with combined serum IgG4 and CA19-9 levels. Pancreatology 2014; 14: 366-372. PMID: 25278306

[20] Sharma C, Eltawil KM, Renfrew PD et al. Advances in diagnosis, treatment and palliation of pancreatic carcinoma: 1990-2010. World J Gastroenterol 2011; 17: 867-897

[21] Kanno A, Masamune A, Hanada K et al. Multicenter study of early pancreatic cancer in Japan. Pancreatology 2018; 18: 61-67. Doi: 10.1016/j.pan.2017.11.007

[22] Ashida R, Tanaka S, Yamanaka $\mathrm{H}$ et al. The role of transabdominal ultrasound in the diagnosis of early stage pancreatic cancer: Review and Single-Center Experience. Diagnostics (Basel) 2018; 9: 2. Doi: 10.3390/diagnostics9010002. PMID: 30587766

[23] D’Onofrio M, De Robertis R, Crosara S et al. Acoustic radiation force impulse with shear wave speed quantification of pancreatic masses: A prospective study. Pancreatology 2016; 16: 106-109

[24] Park MK, Jo J, Kwon $\mathrm{H}$ et al. Usefulness of acoustic radiation force impulse elastography in the differential diagnosis of benign and malignant solid pancreatic lesions. Ultrasonography 2014; 33: 26-33. Doi: $10.14366 /$ usg. 13017

[25] Furuhashi N, Suzuki K, Sakurai Y et al. Differentiation of focal-type autoimmune pancreatitis from pancreatic carcinoma: assessment by multiphase contrast-enhanced CT. Eur Radiol 2015; 25: 1366-1374. PMID: 25433412

[26] Held L. A nomogram for P values. BMC Med Res Methodol 2010; 10 : 21. PMID: 20233437

[27] Zaheer A, Singh VK, Akshintala VS et al. Differentiating autoimmune pancreatitis from pancreatic adenocarcinoma using dual-phase computed tomography. J Comput Assist Tomogr 2014; 38: 146-155. PMID: 24424563

[28] Zhang J, lia G, Zuo C et al. 18F- FDG PET/CT helps differentiate autoimmune pancreatitis from pancreatic cancer. BMC Cancer 2017; 17: 695. PMID: 29061130

[29] Matsumoto I, Shirakawa S, Shinzeki M et al. 18-Fluorodeoxyglucose positron emission tomography does not aid in diagnosis of pancreatic ductal adenocarcinoma. Clin Gastroenterol Hepatol 2013; 11: 712-718. PMID: 23353642

[30] Gu X, Liu R. Application of 18F-FDG PET/CT combined with carbohydrate antigen 19-9 for differentiating pancreatic carcinoma from chronic mass-forming pancreatitis in Chinese elderly. Clin Interv Aging 2016; 11: 1365-1370. PMID: 27729779

[31] Kwon JH, Kim JH, Kim SY et al. Differentiating focal autoimmune pancreatitis and pancreatic ductal adenocarcinoma: contrast-enhanced MRI with special emphasis on the arterial phase. Eur Radiol 2019; 29: 5763-5771. PMID: 31028441

[32] Sun GF, Zuo C], Shao CW et al. Focal autoimmune pancreatitis: radiological characteristics help to distinguish from pancreatic cancer. World J Gastroenterol 2013; 19: 3634-3641. PMID: 23801866
[33] Jang KM, Kim SH, Kim YK et al. Missed pancreatic ductal adenocarcinoma: Assessment of early imaging findings on prediagnostic magnetic resonance imaging. Eur J Radiol 2015; 84: 1473-1479. PMID: 26032128

[34] Lee JH, Min JH, Kim YK et al. Usefulness of non-contrast MR imaging in distinguishing pancreatic ductal adenocarcinoma from focal pancreatitis. Clin Imaging 2019; 55: 132-139. PMID: 30818163

[35] De Lisi S, Buscarini E, Arcidiacono PG et al. Endoscopic ultrasonography findings in autoimmune pancreatitis: be aware of the ambiguous features and look for the pivotal ones. JOP 2010; 11: 78-84. PMID: 20065561

[36] Rösch T, Lorenz R, Braig C et al. Endoscopic ultrasound in pancreatic tumor diagnosis. Gastrointest Endosc 1991; 37: 347-352. PMID: 2070987

[37] Dumonceau JM, Deprez PH, Jenssen C et al. Indications, results, and clinical impact of endoscopic ultrasound (EUS)-guided sampling in gastroenterology: European Society of Gastrointestinal Endoscopy (ESGE) Clinical Guideline - Updated January 2017. Endoscopy 2017; 49: 695-714. PMID: 28511234

[38] Chen G, Liu S, Zhao Y et al. Diagnostic accuracy of endoscopic ultrasound-guided fine needle aspiration for pancreatic cancer: a meta-analysis. Pancreatology 2013; 13: 298-304. PMID: 23719604

[39] Klapman JB, Logrono R, Dye CE et al. Clinical impact of on-site cytopathology interpretation on endoscopic ultrasound-guided fine needle aspiration. Am J Gastroenterol 2003; 98: 1289-1294. PMID: 12818271

[40] Conti CB, Cereatti F, Grassia R. Endoscopic ultrasound-guided sampling of solid pancreatic masses: the fine needle aspiration or fine needle biopsy dilemma. Is the best needle yet to come? World J Gastrointest Endosc 2019; 11: 454-471. PMID: 31523377

[41] Levy M], Wiersema M], Chari ST. Chronic pancreatitis: focal pancreatitis or cancer? Is there a role for FNA/biopsy? Autoimmune pancreatitis. Endoscopy. 2006; 38: S30-S35. PMID: 16802220

[42] Grassia R, Imperatore N, Capone P et al. EUS-guided tissue acquisition in chronic pancreatitis: Differential diagnosis between pancreatic cancer and pseudotumoral masses using EUS-FNA or core biopsy. Endoscopic. Ultrasound 2020; 9: 122-129

[43] Dong Y, D'Onofrio M, Hocke M et al. Autoimmune pancreatitis: Imaging features. Endosc Ultrasound 2018; 7: 196-203. PMID: 28836516

[44] Cho MK, Moon SH, Song TJ et al. Contrast-Enhanced Endoscopic Ultrasound for Differentially Diagnosing Autoimmune Pancreatitis and Pancreatic Cancer. Gut Liver 2018; 12: 591-596. PMID: 29699060

[45] Vitali F, Pfeifer L, Janson C et al. Quantitative perfusion analysis in pancreatic contrast enhanced ultrasound (DCE-US): A promising tool for the differentiation between autoimmune pancreatitis and pancreatic cancer. Z Gastroenterol 2015; 53: 1175-1181. PMID 26480053

[46] Dietrich CF, Hocke M. Elastography of the pancreas. Current viewClin Endosc 2019; 52: 533-540. Doi: 10.5946/ce.2018.156

[47] Dyrla P, Gil J, Niemczyk S et al. Elastography in the Diagnosis of Pancreatic Malignancies. Adv Exp Med Biol 2019; 1133: 41-48. PMID:30443726

[48] Okasha HH, Mahdy RE, Elkholy S et al. Endoscopic ultrasound (EUS) elastography and strain ratio, could it help in differentiating malignant from benign pancreatic lesions? Medicine (Baltimore) 2018; 97: e11689. PMID: 30200064

[49] Okasha H, Elkholy S, El-Sayed R et al. Real time endoscopic ultrasound elastography an strain ratio in the diagnosis of solid pancreatic lesions. World J Gastroenterol 2017; 23: 5962-5968. PMID: 28932088

[50] Lu Y, Chen L, Li C et al. Diagnostic utility of endoscopic ultrasonography-elastography in the evaluation of solid pancreatic masses: A meta-analysis and systematic review. Med Ultrason 2017; 19 : 150-158. PMID: 28440348 